\title{
"QUE DÉUS NE SERÀ SERVIT E VOSALTRES E TOTS NOSTRES SOTSMESES NE SERETS BEN REGITS E GOVERNATS". LAS PROPOSICIONES REALES A LAS CORTES VALENCIANAS DURANTE EL REINADO DE ALFONSO EL MAGNÁNIMO
}

\author{
José Antonio Alabau Calle \\ Raquel Madrid Souto \\ Universitat de València
}

\begin{abstract}
Resumen: La proposición realizada por el monarca -o su representante- ante los brazos convocados a cortes marcaba la apertura oficial de las sesiones de la asamblea y solía hacer referencia al estado político del reino y a la necesidad de un buen gobierno por parte del soberano, así como el motivo de la convocatoria. El análisis de las proposiciones reales del rey Alfonso V no sólo nos acercará a su pensamiento político sino que también nos permitirá conocer sus peticiones a los brazos valencianos, peticiones que estarán marcadas en cada caso por la coyuntura política y económica.
\end{abstract}

Palabras clave: Cortes, Reino de Valencia, Alfonso el Magnánimo.

“Que Déus ne serà servit e vosaltres e tots nostres sotsmeses ne serets ben regits e governats”. The royal requests to the Valencian parliament during the reign of Alfonso the Magnanimous

Abstract: The request made by the monarch, or his delegate, to the parliamentary estates called upon the parliament marked the beginning of the official sessions. That proposal used to recall the political status of the kingdom, underscoring the need of a good government by the king, together with expressing the main reason of the gathering. The analysis of the royal proposals made by Alfonso $\mathrm{V}$ will not only allow us to take a close approximation to the sovereign's political thought, but it will also serve as a way to understand his petitions to the Valencian estates. Such petitions will ultimately be affected by the political and economic circumstances of any particular period of time.

Key words: Representative assemblies, Kingdom of Valencia, Alfonso the Magnanimous.

La profesora Rosa Muñoz Pomer ha contribuido con sus numerosos estudios a profundizar en el conocimiento de instituciones valencianas como la Generalitat-gracias a su tesis doctoral- y a establecer el funcionamiento de las asambleas parlamentarias en época medieval. Además, ha sido una de las impulsoras del proyecto de edición de los procesos de las cortes valencianas durante el período foral -iniciado en los años 90- en el que siempre ha demostrado su gran interés por la aplicación de las nuevas tecnologías al campo de la investigación histórica que conllevaran tanto un mejor acceso como una mejor conservación de la documentación.

Data de recepció: 5 de desembre de 2014 / Data d'acceptació: 26 de febrer de 2015. 
Fue durante nuestros estudios de doctorado cuando la profesora Muñoz orientó nuestras investigaciones hacia el estudio de las asambleas parlamentarias medievales, compartiendo con nosotros tanto sus infinitos conocimientos sobre el tema como los resultados de su paciente labor investigadora, guiando nuestros estudios con sus acertados consejos y haciéndonos partícipes del proyecto de edición. Sirva, pues, el presente artículo como agradecimiento y homenaje no sólo por su labor docente e investigadora, sino por su enorme calidad humana.

\section{LA CELEBRACIÓN DE CORTES EN EL REINO DE VALENCIA}

La celebración de cortes por los monarcas de la Corona de Aragón se caracterizaba por un estricto protocolo y un gran despliegue de fastuosidad y esplendor que enaltecía a los reyes sentados en el solio real ante sus súbditos. El emplazamiento elegido para el desarrollo de las sesiones parlamentarias solía ser una iglesia (la catedral de Valencia, la iglesia de Santa María de Traiguera, etc.) o el convento de Predicadores de Valencia, no sólo por los aspectos prácticos que conllevaban esta elección -los brazos podían reunirse por separado en las distintas capillas o celdas de los frailes- sino porque también era una forma de presentar al monarca como líder espiritual de su pueblo (Cortés, 1994, 113-117). Una vez el rey y los brazos militar, eclesiástico y real ocupaban el lugar que por protocolo les correspondía, la apertura oficial de las cortes se realizaba con la solemne lectura de la proposición real realizada por el monarca -o su representante- y donde normalmente se hacía referencia a la necesidad del buen gobierno, al estado político del reino y se exponía el objeto de la convocatoria (Albert y Gassiot, 1928, 9-14). Dado el ceremonial empleado y el ambiente sagrado en el que se desarrollaban estas asambleas, el discurso real llegó a copiar la estructura del sermón religioso utilizada por los predicadores, componiéndose piezas que brillan por su erudición y solemnidad, destacando los pertenecientes a Pedro el Ceremonioso y su hijo Martín el Humano (Cawsey, 2008, 173-193; Cátedra, 1985-1986, 17-47). Con el advenimiento de la dinastía Trastámara, el uso del sermón político decae, las proposiciones reales pasan a ser en la mayoría de los casos breves y formularias, pero no por ello pierden su interés como fuente de información sobre el pensamiento político de la monarquía, revelándonos su análisis aspectos sobre la concepción del poder real y su justificación.

\section{ALFONSO EL MAGNÁNIMO Y LAS CORTES VALENCIANAS ${ }^{1}$}

La política de expansión mediterránea iniciada por los monarcas de la Corona de Aragón en el siglo XIII no fue abandonada por la dinastía Trastámara entronizada tras el Compromiso de Caspe, convirtiéndose en uno de los objetivos principales de Alfonso V tras su acceso al trono en 1416. El deficiente estado de las arcas reales y la necesidad de financiación de las empresas italianas llevarán al Magnánimo a convocar cortes como una de las formas de obtener préstamos y subsidios para poder llevar a cabo su política medi-

\footnotetext{
1 Agradecemos a las profesoras Josepa Cortés Escrivà, Ma Rosa Muñoz Pomer y Mª José Carbonell Boria que nos facilitaran en su día la transcripción inédita de las cortes de 1417, 1419 y 1429 respectivamente para los distintos trabajos de investigación que nos hallamos realizando.
} 
terránea. Es por ello que hasta en ocho ocasiones los tres brazos valencianos fueron convocados por el monarca o sus lugartenientes -su mujer, la reina María, y su hermano Juan-, siendo en cinco de ellas la necesidad de financiación de las campañas italianas el motivo de la convocatoria $(1419,1421,1435,1436$ y 1443). Otras causas para la reunión de las cortes serán el juramento de los fueros por el monarca al acceder al trono (1417), la reforma de la Diputación del General (1428) y la guerra contra Castilla (1429).

Uno de los cambios introducidos por los Trastámara en el que los historiadores han hecho un mayor hincapié es en el uso del castellano en los discursos de apertura de las reuniones parlamentarias. Efectivamente, Alfonso utilizó en la mayoría de las ocasiones el castellano en sus discursos (1417, 1428 y 1429), únicamente en las cortes de 1419 se dirigió a los brazos en valenciano. En el caso de sus lugartenientes, la reina María alternará en sus discursos el uso del castellano $(1421,1435)$ y el valenciano (1443), mientras que la única vez que Juan se encargó de la apertura de las cortes durante la ausencia de Alfonso (1437), también se decantará por el uso del valenciano.

Otra de las características que diferencia notablemente la oratoria real del reinado del Magnánimo de la del período precedente, es la extrema brevedad de los discursos, abandonándose la estructura del sermón escolástico plagado de citas bíblicas y de auctoritates clásicas como Valerio Máximo, Salustio o Cicerón, para centrarse en la necesidad del bienestar del reino y su buen gobierno. De esta forma, el rey introducía de forma rápida y clara la petición de ayuda a los brazos, que se traducía siempre en la concesión de subsidios y donativos que le permitieran seguir adelante con sus proyectos de expansión. Asimismo, encontramos de forma habitual en los discursos tanto del Magnánimo como de la reina María y de Juan de Navarra, no sólo el recuerdo a los monarcas anteriores -de cuyo linaje desciende Alfonso, legitimando así la elección de Caspe- sino también la profunda estima que éste profesa a sus súbditos, que son gobernados por él con la ayuda de Dios, a modo de preámbulo protocolario y de cortesía, previo a la exposición de la petición real. Para época Moderna, Emilia Salvador ha señalado la persistencia de este tipo de discurso, calificándolo como "atemporal", en el que solía darse muestra de "el amor del rey a sus súbditos, la fidelidad de estos hacia su rey, el deseo de éste de proceder a la recta administración de la justicia y buen gobierno, etc. etc.", (Salvador, 1998, 396). El discurso ante las cortes también es aprovechado por el monarca para legitimar a sus lugartenientes que, durante sus ausencias del reino, debían sustituirlo en las funciones de gobierno:

Certifficant-vos que nós, ans de nostra partida, ab la ajuda de Déu, lexarem en tal regiment nostres regnes e terres durant nostra absència, que Déus ne serà servit e vosaltres e tots nostres sotsmeses ne serets ben regits e governats (ARV, RC, $511, \mathrm{f} .7 \mathrm{v})$.

La respuesta de los brazos a la proposición real era realizada normalmente por un representante del brazo eclesiástico -el obispo de Valencia o el maestre de la Orden de Montesa- que en nombre de todos los convocados, solía posponer la respuesta definitiva a las peticiones reales hasta el momento en que se hubieran llevado a cabo las deliberaciones de los brazos y resuelto los agravios presentados ante el monarca. También se trata de discursos en extremo breves y de cortesía, que no copian la estructura del sermón, a pesar de ser realizados por eclesiásticos. Sólo en una ocasión encontramos una cita bíblica: se trata de la respuesta dada al rey por frey Romeu de Corbera, maestre de la Orden de Montesa, en las cortes de Valencia de 1428, que cita el Libro de los Reyes, atribuyendo las 
mismas cualidades del rey Salomón al monarca (AMV, PC, yy-10, f. 25v). Esto no ocurre en Aragón y Cataluña, donde los brazos responden a la proposición real con elaborados discursos -ya sean en latín como en aragonés o catalán-, salpicados de citas bíblicas y de autores clásicos, continuándose con la tradición sermonaria anterior.

\section{Las cortes de 1417-1418}

La primera ocasión en la que el Magnánimo pronunció un discurso ante las cortes valencianas fue con motivo del juramento de los fueros y privilegios del reino al acceder al trono. En el discurso, escrito en castellano y leído en persona por el propio rey Alfonso, el monarca recordó que ya con anterioridad había jurado los fueros y privilegios "en tiempo que éramos primogénito" durante las Cortes celebradas por su padre, el rey Fernando I, en la ciudad de Valencia entre 1413 y 1415 (AMV, PC, yy-6, f. 396r), si bien a instancias de la ciudad de Valencia, volvía a realizarlo ahora ya como rey. Tras ello, el Magnánimo continuó su discurso con las habituales referencias al buen gobierno y a su intención de

fer algunas cosas tocantes servicio de Dios, honor nuestra e bien avenir d'aquesto reyno, el qual havemos en singular reputación por los notables e grandes servicios que ha feytos a la corona d'Aragón (AMV, PC, yy-8, f. 18r).

El encargado de responder al monarca en nombre de los tres estamentos del reino fue el infante Juan de Aragón, hermano menor del rey quien, de modo protocolario y cortés, aplazó cualquier decisión a futuras deliberaciones de los brazos. Como hemos apuntado con anterioridad, lo habitual era que la respuesta a la proposición real recayera en manos de algún miembro destacado del brazo eclesiástico, preferentemente el obispo de la diócesis de Valencia. En esta ocasión, teniendo en cuenta la ausencia de miembros destacados del estamento eclesiástico en el momento de la proposición inicial del Magnánimo (ARV, RC, 509, ff. 1-12v), la representación de los brazos del reino recayó en el infante Juan de Aragón, hermano del rey, como cabeza del brazo militar. Además, debemos tener presente que, como infante de Aragón, mantenía posibilidades reales de suceder en el trono a su hermano, pues cabe recordar que el Magnánimo todavía no tenía herederos.

Días después, y una vez solventado el trámite del juramento de fueros, privilegios y fidelidad al nuevo rey, Alfonso el Magnánimo realizó una nueva propuesta ante las cortes. En esta ocasión, tras las habituales referencias a la paz, la concordia y al buen gobierno del reino, y manifestar su intención de solventar cualquier agravio que él, sus oficiales o los predecesores de ambos hubieran podido causar a los regnícolas, el Magnánimo alegó la pésima situación en la que había encontrado las finanzas reales como motivo para solicitar la ayuda económica del reino:

com en nostre novell regiment hajam trobat que totes les rendes del dit regne quasi són alienades en tant que nós bonament no podem provehir a sostenir nostre real sta(t), ni altres coses fer a què som tenguts per nostra real dignitat (AMV, PC, yy-8, f. 50r).

Además, instó a los estamentos del reino a nombrar tractadors para que le pudieran presentar los agravios que consideraran oportunos, y poder así avanzar en el desarrollo de las cortes. 
En esta ocasión la respuesta al monarca quedó en manos del obispo de Valencia, Hug de Llupià, quien en nombre de los tres estamentos manifestó al rey la intención y voluntad de "servir de vostra senyoria e bé e profit de la cosa pública d'aquest regne" (AMV, PC, yy-8, f. 50r). Tal y como hemos señalado anteriormente, este tipo de respuesta era la habitual en las cortes valencianas, pues el acuerdo definitivo respecto al donativo a conceder al monarca solía producirse tras la resolución de los agravios presentados por los estamentos del reino.

\section{Las cortes de 1419}

Las cortes celebradas en Valencia en 1419 tuvieron como escenario el palacio episcopal y como contexto la partida del Magnánimo a Cerdeña y Sicilia, dentro de la política expansionista mediterránea del monarca aragonés. El discurso del rey Alfonso tuvo lugar el 29 de agosto, sólo un día después de la fecha de apertura de las cortes fijada en la convocatoria. Se trata de la única vez en que el monarca realizó un discurso en valenciano. En primer lugar, el Magnánimo explicó a los estamentos del reino el motivo de su convocatoria:

Havem deliberat, ab la ajuda de nostre senyor Déu, visitar personalment los nostres regnes de Sicília e de Cerdenya, e per ço havem feta armada de certes galeas e altres fustes, $e$, ans de nostra partida, havem convocat a vosaltres per notificar-vos les dites coses $e$ pendre comiat de vosaltres, dels quals nos tenim notablement per servits, de què.us som obligats proseguir-vos de gràcies e favors.

Acto seguido, alegando el elevado gasto realizado en la construcción y preparativos de la armada que le había de acompañar en su viaje, el rey expresó la necesidad de que las cortes le concedieran algún tipo de ayuda económica:

Hajam necessàries algunes peccúnies, per ço, recorrents a vosaltres, qui en nostres necessitats tots temps per vostra innada naturalesa e liberelitat nos havets soccorregut e ajudat (ARV, RC, 511, f. 7r).

Para asegurar, seguidamente, que los estamentos del reino no debían temer por el gobierno durante su ausencia, pues antes de su partida se había asegurado de que todo quedase arreglado para que sus territorios fuesen gobernados debidamente durante su ausencia.

Una vez más, la respuesta a la proposición real corrió a cargo de Hug de Llupià, obispo de Valencia, como representante de los tres estamentos. Con la cortesía preceptiva en estos casos, el obispo Llupià mostró al rey la alegría de los tres brazos por su presencia, pero remitió al monarca a la posterior deliberación de los estamentos del reino para poder ofrecer una respuesta detallada a su proposición (ARV, RC, 511, f. 7v). Contrariamente a lo acostumbrado, los brazos no esperaron a la resolución de los agravios para responder debidamente a la proposición del Magnánimo, ya que ésta no se limitaba a la habitual petición pecuniaria, sino que además incluía la intención del monarca de abandonar sus reinos peninsulares. De este modo, transcurridos apenas cuatro días desde el discurso del monarca, los tres estamentos del reino, representados de nuevo por el obispo Hug de Llupià, mostraron al Magnánimo su disconformidad con el viaje que pretendía emprender, solicitándole que abandonase dicha idea. El obispo de Valencia justificó la postura de los brazos del reino a partir de cuatro consideraciones principales: 
$1^{\text {a) }}$ que el viaje era totalmente innecesario:

com vós, senyor, ni vostres regnes e terres que havets deçà mar no siats ne sien en tal punt o stament que la vostra reyal persona se deja partir de aquelles.

$2^{\mathrm{a})}$ la falta de un heredero al trono:

que encara a Déu no ha plagut donar a vós primogènit qui aprés vostres benaventurats dies puxa succehir e regnar en vostres regnes e terres.

$3^{\text {a) }}$ la situación prebélica en las fronteras de la Corona:

que en alguns dels regnes e terres circumvehins ha molta gent d'armes ajustada, per les quals a vostres regnes se poria en vostra absència inconvenients e sinistres seguir

$4^{a}$ ) la peligrosidad de navegar en ese periodo del año:

lo temps en què som ja no és dispost per navegar, senyaladament vostra reyal persona (ARV, RC, 511, f. 15r).

Ante la postura mostrada por los tres estamentos, el rey Alfonso desplegó su capacidad oratoria, contrarrestando brillantemente los argumentos anteriores. En primer lugar, y con la cortesía propia de la retórica del Magnánimo, el rey agradeció la preocupación mostrada por sus vasallos, preocupación que, según el propio monarca, nacía de

La feel e notable intenció e amor que tots temps havets haut e mostrada a nostres predecessors e no menys a nós.

Acto seguido, procedió a desmontar argumento por argumento las consideraciones expuestas por los estamentos. Ante la consideración de que el viaje planteado por el monarca fuera totalmente innecesario, el rey Alfonso esgrimió que se trataba de una cuestión de honor:

Per satisfer a nostra honor nafrada, (...) aquell no poríem jaquir sens gran càrrech $e$ desonor nostra.

Respecto a la falta de heredero que garantizara su sucesión, el Magnánimo contraargumentó alegando que eso era algo que quedaba en manos de Dios y que, por tanto, no quedaba otro remedio que aceptar lo que éste dispusiera al respecto:

açò stà en la ma e voler de nostre senyor Déu, lo qual si serà nós servir el nos lo darà e, si no, serem contents de sa ordinació e voler.

Sobre la situación prebélica en las fronteras de la Corona, el Trastámara restó importancia a la posibilidad de que sus enemigos aprovechasen su ausencia, pues ya en el pasado se habían dado situaciones semejantes, solventándose sin mayores dificultades: 
crehem que aquesta gent ha prou a fer en deffensar-se que hajen poch voler e menys manera de moure a nós guerra en nostre regne, en special com los haya mal pres altres vegades en semblants moviments, axí en presència com en absència de nostres predecessors.

Respecto a los peligros de la navegación a esas alturas del año -septiembre-, el Magnánimo alegó que podía navegar de jornada en jornada a través de sus posesiones, mostrando total confianza en que Dios tendría a bien ofrecerle buen tiempo, tal y como había hecho con sus antecesores en diversas ocasiones y en peores épocas del año:

quasi de jornada en jornada podem anar per nostras illas, e que nostre Senyor té lo bon temps en sa mà per aquells que vol endreçar, que axí com ha endreçat e guiat a nostres predecessors de gloriosa memòria qui en tan avançat temps e més, moltes vegades se són recullits e han fets viatges gloriosament, confiam per sa misericòrdia endreçarà e guiarà a nós.

Contrarrestados los argumentos empleados por los estamentos, el rey Alfonso terminó su discurso aprovechando el último de ellos en favor propio, al instar a las cortes a que aceleraran la marcha de las reuniones evitando así que empeorase el tiempo y, en consecuencia, aumentasen las dificultades de la navegación:

Us pregam que per no perdre del bon temps que.ns resta, vos desempechets breument perquè d'aquell prenam la major part que puscam (ARV, RC, 511, f. 16r).

\section{Las cortes de 1421}

Las cortes de Traiguera-Las Cuevas-San Mateo de 1421 son las primeras presididas por un lugarteniente general del rey Alfonso con motivo de su presencia en territorios italianos. La reina María, esposa del Magnánimo, será la encargada de realizar la proposición ante los estamentos reunidos en la iglesia de Santa María de la villa de Traiguera, si bien no fue ella personalmente la que pronunció el discurso, sino que fue leído por Raimón Batle, lugarteniente del protonotario real.

El discurso de la reina María se inició con la exposición de los motivos que la habían llevado a convocar cortes en el reino de Valencia, a pesar de que dicha prerrogativa quedaba reservada por fueros a las figuras del rey y su primogénito. Ante la ausencia del Magnánimo, y la reciente retirada de éste a Cerdeña abandonando el asedio de Bonifacio, la reina María había decidido convocar al consell real. Entre otras cuestiones, dicho consejo trató de la conveniencia de convocar cortes generales de la Corona de Aragón, tal y como le había sugerido a la reina la ciudad de Barcelona mediante el envío de mensajeros a Tortosa, ciudad en la que estaba reunida con su consejo. Siguiendo la opinión de gran parte del consejo real y de la ciudad de Valencia, la reina decidió:

que los del regne d'Aragó fóssen convocats en cert loch dins lo dit regne d'Aragó e los del regne de València en cert loch dins aquell dit regne de València. E los del principat de Catalunya en cert loch dins lo dit principat (ACA, Cancillería, PC, 29, ff. 6r-6v). 
Tras explicar las razones de la convocatoria, la esposa del Magnánimo rogó a los estamentos del reino de Valencia que, a pesar de la legislación contraria, tuvieran a bien aceptar la convocatoria, pues no era su intención perjudicar las libertades del reino ni alegar en el futuro la excepción concedida en esta ocasión:

no obstants los dits furs e privilegis consintats en la dita convocació e celebració e en abilitació de aquella [...] no contrestants los dits furs e privilegis als quals per la convocació no sia en res prejudicat ne derogat. Ans aquells romanguen en sa força e valor (ACA, Cancillería, PC, 29, f. 6v).

La respuesta a la proposición de la reina María corrió a cargo de Joan Gascó, doctor en leyes quien, en nombre de los tres estamentos del reino, manifestó el consentimiento de la corte a la convocatoria efectuada por la lugarteniente del Magnánimo, principalmente atendiendo a la excepcional situación del reino ante la ausencia del monarca y la falta de primogénito que ocupase su lugar, así como a la intención manifiesta de la reina de que la legalidad foral no se viese perjudicada en el futuro:

attesa la absència del dit senyor rey e considerats que l dit senyor rey a present no ha primogènit lo qual puxa supplir en los dits affers. E attés que vós, senyora, axí com a lochtinent del dit senyor havets convocat la dita cort principalment per demanar consell en los dits affers, sens derogació dels dits furs e privilegis.

\section{Las cortes de 1428}

Con motivo de la grave situación económica que atravesaba la Diputació del General en el reino de Valencia en 1428, el rey Alfonso V volvió a convocar a los estamentos del reino a principios de dicho año. Las cortes debían inaugurarse el 20 de febrero, y el lugar escogido para su celebración fue el convento de los Predicadores de la ciudad de Valencia, si bien el discurso del monarca no se produjo hasta casi dos meses después, en concreto el 14 de abril. Con el regreso del rey Alfonso a las cortes tras su aventura italiana, volvemos a encontrarnos un discurso elaborado, realizado en castellano, que -sin llegar a la complejidad de los discursos de monarcas anteriores- sí es más sofisticado que el pronunciado ante los brazos en 1421 por la reina María. Como venía siendo la tónica habitual en los discursos del Magnánimo, éste comenzó con un reconocimiento a los servicios prestados por sus vasallos para, a continuación, explicar el motivo de la convocatoria de la asamblea: la mala situación económica de la Generalitat.

Los drechos de les generalidades del dicho Regno son tanto diminuidos que a penas bastan a les pensiones annuales e cargas de las dichas generalidades.

Ante esta situación, el rey Alfonso solicitó a los estamentos del reino

que con consello e ayuda vuestros pueda proveir saludablemente en lo sobredito e encara en otras cosas. 
El monarca recibió respuesta a su proposición de boca de Romeu de Corbera, maestre de la Orden de Santa María de Montesa quien, debido a la vacante de la sede episcopal valentina, asumió la representación de los estamentos del reino como cabeza del brazo eclesiástico. Tal y como hiciera en las cortes presididas por Fernando I en 1413-1415 (AMV, PC, yy-9, f. 229v), el maestre de Montesa estructuró su respuesta a partir de una cita bíblica, en concreto el pasaje del Libro de los Reyes (1Reyes 10,8) en el que la reina de Saba se dirige al rey Salomón con las siguientes palabras:

Benahuyrats son los teus pobles, e benahuyrats son los teus servents e tots aquells qui davant tu stan continuament oynt la tua saviesa e doctrina.

Tras atribuir al rey Alfonso las virtudes del rey Salomón, el maestre de Montesa continuó las adulaciones al Trastámara destacando el amor que le profesaban sus vasallos debido a su buen gobierno y deseando que Dios le concediera una larga vida, para terminar, como era habitual, emplazando al Magnánimo a posteriores deliberaciones de los estamentos para poder obtener una respuesta a su proposición.

\section{Las cortes de 1429}

Con motivo de la guerra con Castilla, el 15 de noviembre de 1429 el rey Alfonso el Magnánimo pronunció en persona el último de sus discursos, la última de sus proposiciones ante las cortes del reino de Valencia pues, a finales de mayo de 1432, partió hacia tierras italianas, donde encontraría la muerte el 27 de junio de 1458 sin haber regresado nunca más a la península (Ryder, 1992, 228; 524).

El rey, que pronunció su discurso en castellano, inició la proposición ante los representantes de los tres estamentos reunidos ante su persona en la iglesia de Santa María de Traiguera, explicando los motivos que le habían llevado al enfrentamiento con su primo Juan II de Castilla. Como puede apreciarse, el objetivo del Magnánimo fue mostrarse como víctima del conflicto que mantenía con el monarca castellano, a quien acusó de haber sido el único causante de la guerra que enfrentaba a ambas coronas.

como el rey de Castiella, segunt dito es, sin alguna justa causa e contra tota justicia e rahon ha indicta e movida guerra contra nós e nostros regnos e tierras, e con todo su poder de gent d'armas se sforçe entrar dentro el regno d'Aragón [...] con intencion de destroir nostros regnos e tierras (AMV, PC, yy-12, f. 12r).

El rey Alfonso no quería aparecer ante sus vasallos como el causante del conflicto, así que incluso justificó la respuesta a la agresión armada castellana recurriendo a su dañado honor.

E aprés nós no quiriendo fazer ni dar todo el danyo que podiamos el dito rey de Castiella, mas por suplir a nostra honor, entramos en el regno de Castiello e prendiemos alguna partida de su tierra (AMV, PC, yy-12, f. 12v).

Continuó su discurso advirtiendo a los tres estamentos del reino del peligro de una nueva invasión castellana, especialmente para la seguridad e integridad del reino de Valencia, dado que el rey de Castilla estaba preparando un nuevo ataque: 
segunt sabemos de cierto el dito rey de Castiella se prepara lo mas poderosament que puede por entrar a invadir e daminificar nostros regnos e tierras, senyaladament aquesti regno de Valencia.

Para finalizar, el Magnánimo solicitó la ayuda del reino para poder defenderlo debidamente ante la posibilidad de un inminente ataque castellano:

rogamos que breument querades en tal manera nos ayudar que nos mediant la gracia divinal podamos [...] el regno de Valencia e los regnícoles de aquell preservar e deffender.

El encargado de responder a la proposición real en nombre de los tres estamentos del reino fue el recientemente nombrado obispo de Valencia, Alfonso de Borja.

sobre les coses proposades per vostra altesa, los dits tres staments praticaran e parlaran entre sí. E hauda delliberació vos faram tal resposta que serà servey de Déu e exalçament de la vostra corona e benefici, e repòs de la cosa pública del dit regne e extermini encara de vostres enemichs (AMV, PC, yy-12, f. 12v).

\section{Las cortes de 1437-1438}

Las cortes celebradas entre febrero de 1437 y mayo de 1438 en la ciudad de Valencia fueron, en gran parte, consecuencia de las cortes generales de Monzón de 1436 convocadas por la reina María al conocer la captura del rey Alfonso tras el desastre de Ponza (Ryder, 1992, 267). Al licenciar las cortes de Monzón, se había llegado al acuerdo de que se debían convocar cortes particulares para cada reino de la Corona con el objetivo de solventar las numerosas dificultades que habían llevado a punto muerto la reunión en la villa aragonesa. Si bien en Aragón las cortes particulares se iniciaron prácticamente sin solución de continuidad, en el caso valenciano hubo que esperar hasta febrero de 1437 para que se celebrasen cortes particulares.

Ante la ausencia del rey Alfonso, que como sabemos se encontraba inmerso en la conquista de Nápoles, las cortes del reino de Valencia fueron convocadas en diciembre de 1436 por su hermano Juan, rey de Navarra, y lugarteniente general del Magnánimo. Aunque la convocatoria fijaba como fecha de inicio el 15 de enero de 1437 , no fue hasta el 21 de febrero de 1437 cuando el lugarteniente Juan dirigió su discurso a los estamentos del reino reunidos en la catedral de la ciudad de Valencia.

En la primera parte del discurso, redactado en valenciano, y pronunciado por el secretario real Antoni Noguera, el hermano del Magnánimo recordó las cortes generales de Monzón, en las que la reina María, primero, y el propio lugarteniente Juan, después, habían reunido a todos los reinos deçà la mar, incluido el reino de Mallorca. A continuación, prosiguió su discurso justificando el hecho de que hubiese expirado la convocatoria de cortes en Morella previstas para junio de 1436 (ARV, RC, 236, f. 2v), lugar en el que los estamentos del reino de Valencia debían haber continuado de manera individual las cortes de Monzón, según lo acordado en el momento en que la reina María las licenció. El motivo de que las cortes previstas en Morella no se hubiesen realizado debía buscarse en las largas y delicadas negociaciones que el lugarteniente, en nombre del Magnánimo, había tenido que mantener con los castellanos para firmar la paz con Juan II de Castilla, amén 
de otros asuntos de primer orden que requerían su presencia y que le imposibilitaron acudir a Morella en el plazo previsto:

Per ço, com dins lo temps statuït no·y poguem ésser, entenents en la bona conclusió de la pau, mijançant la gràcia del Sanct Spírit feta e fermada entre lo senyor rey e nós, d'una part, e lo rey de Castella, de l'altra, e en altres affers concernents honor e servey del dit senyor rey, frare nostre, los quals necessàriament requiren nostre presència (AMV, PC, yy-15, f. 51r).

Tras este pequeño preámbulo, el rey Juan de Navarra solicitó la ayuda y socorro del reino de Valencia, ofreciéndose a reformar la justicia y aquellos aspectos que impulsaran el buen gobierno del reino.

La respuesta a la proposición realizada por el lugarteniente Juan de Navarra corrió a manos del máximo representante del brazo eclesiástico en el reino de Valencia, Alfonso de Borja, obispo de Valencia, quien, tal y como había hecho el hermano del Magnánimo, no pronunció su discurso personalmente, sino que pidió al secretario Antoni Noguera que lo hiciera por él. La postura mostrada por los estamentos a la proposición real fue de abierto rechazo, dado que la convocatoria de cortes era prerrogativa del rey o, en caso de necesidad, de su primogénito, y ninguna de estas condiciones se daban en ese caso, por lo que la convocatoria del lugarteniente general era contraria a derecho y, por consiguiente, todo lo derivado de la misma también lo era:

la dita que's diu convocació, per consegüent, les dites que $s$ dien prorogacions, per medi de les quals se ve a celebració de corts generals, no podien, pogueren ne poden ésser fetes, sino per lo dit senyor rey, o en cas d'urgent necessitat per son primogènit (AMV, PC, yy-15, f. 52r).

Tras ello, el obispo de Valencia solicitó que todas las protestas presentadas al respecto por los brazos durante las numerosas prórrogas que se habían sucedido hasta la llegada del hermano del Magnánimo, las tuviera éste como presentadas ante su persona, y que desistiese de pretender iniciar las cortes.

Ante la postura de los estamentos, al lugarteniente Juan no le quedó más remedio que prorrogar las cortes y entablar negociaciones paralelas con los brazos del reino para conseguir su apoyo y que las cortes pudiesen iniciarse, especialmente con la ciudad de Valencia (AMV, PC, yy-16, f. 1r). Así, finalmente, el 19 de marzo de 1437, transcurrido poco más de un mes desde que el lugarteniente general realizara su proposición a los brazos del reino, éstos le habilitaron para convocar y presidir las cortes, no sin antes asegurarse de que la legalidad foral no iba a verse afectada por este hecho (AMV, PC, yy-15, f. 75v).

\section{Las cortes de 1443-1446}

Las últimas cortes del reinado de Alfonso el Magnánimo en el reino de Valencia se celebraron entre el 16 de marzo de 1443 y el 6 de julio de 1446. Su esposa, la reina María, fue la encargada de convocar y presidirlas en calidad de lugarteniente general del Magnánimo. En su discurso, pronunciado en valenciano, la reina manifestó a los miembros de los estamentos que había convocado dichas cortes en vistas al regreso de su esposo al rei- 
no de Valencia, pues el mismo monarca le había manifestado en diversas ocasiones que esa era su intención y deseo:

Com nós siam certa per letres, instruccions e en altra manera del bon voler e la indisposició que $\cdot$ l dit senyor ha de present de venir e tornar en sos regnes e terres e senyaladament en aquest regne de València (AMV, PC, yy-17, f. 26v).

Ante esta situación -la próxima venida del rey, que sabemos que nunca llegó a producirse-, la reina terminó su discurso exhortando a los miembros de los brazos eclesiástico, militar y real a que no demoraran en exceso la elección de tratadores de agravios para que, de esta manera, las cortes pudieran continuar a buen ritmo y licenciarse con prontitud.

Se volvía a repetir la situación vivida en las cortes de 1437-1438, cuando el por entonces lugarteniente general Juan de Navarra, hermano del Magnánimo, convocó y presidió las cortes del reino. Recordemos que la reacción de los estamentos en dicha ocasión fue de enfrentamiento con el lugarteniente, al que acusaron de no tener capacidad para convocar cortes ni presidirlas, pues estas eran funciones reservadas al monarca o, en su caso, al heredero, según marcaba la legislación foral. En esta ocasión la actitud de los brazos del reino fue similar, pues ya incluso antes de que la reina María realizase su proposición, los estamentos le habían advertido que desistiese de ello, atendiendo a lo establecido por los fueros (AMV, PC, yy-17, f. 26r). Los estamentos respondieron a la proposición de la reina María por boca del maestre de Montesa, Romeu de Corbera, quien con un lenguaje protocolario y según lo habitual, comunicó a la reina que la respuesta definitiva de los estamentos a su proposición la tendría una vez se hubieran reunido y hubieran llegado a un acuerdo entre ellos. Como era de esperar, las protestas de los estamentos del reino ante la convocatoria de la reina María continuaron, tanto conjuntamente como por separado. Finalmente, tras casi un mes de protestas por parte de los brazos y acusaciones de contumacia por parte de la corona, los estamentos aceptaron la convocatoria, proposición y presidencia de las cortes por parte de la reina María, atendiendo a la ausencia del rey Alfonso y a su intención manifestada de regresar a Valencia y salvaguardando, como era habitual en estos casos, la legalidad foral, dejando patente la excepcionalidad del caso.

\section{CONCLUSIONES}

Dado que este trabajo es una primera aproximación a las proposiciones reales del reinado de Alfonso el Magnánimo, gran parte de las conclusiones que aquí podemos señalar son provisionales, a la espera de poder analizar en sucesivas investigaciones los discursos del rey Alfonso y sus lugartenientes ante las cortes de los restantes territorios de la Corona.

Teniendo en cuenta lo anterior, nuestras primeras conclusiones son:

a) Las proposiciones en cortes durante el reinado del Magnánimo son una buena fotografía de la situación política y económica del reino de Valencia en el momento en que se celebraron cada una de las reuniones.

b) Durante las cortes presididas por el rey Alfonso, no encontramos discursos excesivamente elaborados y solemnes, tal y como los podemos encontrar en el caso de las proposiciones de Pedro el Ceremonioso o Martín el Humano, sino proposiciones articuladas de manera clara y sencilla. 
c) En lo que respecta a las reuniones presididas personalmente por el Magnánimo, la actitud habitual de los estamentos ante los planteamientos del monarca fue de colaboración.

d) El primer enfrentamiento entre el monarca y los estamentos ante la proposición real surgió a raíz de que éste manifestase su intención de abandonar la península para iniciar sus capañas italianas (Córcega y Cerdeña primero, Nápoles después).

e) La falta de heredero al trono influyó considerablemente en la postura mantenida por los estamentos del reino.

f) La política mediterránea del Magnánimo aumentó el protagonismo de la figura del lugarteniente, quien en ausencia del monarca y a falta de heredero, debía convocar y presidir las reuniones aunque ello no se ajustara a la legalidad foral.

g) Las cortes de 1437-1438 y las de 1443-1446 suponen el momento de mayor enfrentamiento entre los estamentos y la corona, al atribuirse los lugartenientes prerrogativas que únicamente correspondían al rey o a su heredero.

\section{BIBLIOGRAFÍA}

ALBERT, R. y GASSIOT, J. (1928): Parlaments a les Corts Catalanes (Els nostres clàssics XIX$\mathrm{XX)}$, Barcelona, Barcino.

CÁTEDRA, P. M. (1985-1986): “Acerca del sermón político en la España medieval (A propósito del discurso de Martín el Humano en las cortes de Zaragoza de 1398)", Boletín de la Real Academia de Buenas Letras de Barcelona, XL, 17-47.

CAWSEY, S. F. (2008): Reialesa i propaganda. L'eloqüència reial i la Corona d'Aragó, c. 12001450, Valencia, Publicacions de la Universitat de València.

CORTÉS ESCRIVÁ, J. (1994): "El discurso de la corona”, en Pinilla Pérez de Tudela, R. (ed.): Las Cortes Forales Valencianas: Poder y Representación, Valencia, Corts Valencianes, 113-117.

MUÑOZ POMER, M. R. (1987): Orígenes de la Generalidad Valenciana, Valencia, Conselleria de Cultura, Educació i Ciència, Generalitat Valenciana.

RYDER, A. (1992): Alfonso el Magnánimo. Rey de Aragón, Nápoles y Sicilia (1396-1458), Valencia, Edicions Alfons el Magnànim.

SALVADOR ESTEBAN, E. (1988): "Los discursos de la corona en las cortes de Monzón durante el reinado de Carlos I. Atemporalismo y crónica”, Studia Histórica. Historia Moderna, 6, 381-397. 
